\title{
CONSIDERACIONES SOBRE LA UTILIZACIÓN DE DIFERENTES DENSIDADES EN EL CULTIVO DE PAPAYA (Carica papaya, L.) "BAIXINHO DE SANTA AMALIA" EN ISLAS CANARIAS ${ }^{1}$
}

\author{
$\mathrm{M}^{\mathrm{a}}$ CRISTINA RODRIGUEZ PASTOR ${ }^{2}$
}

\begin{abstract}
RESUMEN.- El cultivo de papaya en Islas Canarias se ha extendido en los últimos años bajo condiciones de invernadero. La utilización de cultivares de papaya tipo "Baixinho de Santa Amalia" (mutante natural del cultivar "Sunrise") de porte bajo, emisión de la flor a corta altura y precocidad en la floración, resultan de gran interés sobre todo en esta clase de medios. Estas características fenológicas hacen posible el manejo del cultivo a mayores densidades que las empleadas con otros cultivares. Por lo tanto, se ha planteado este trabajo cuyo objetivo principal es determinar cual es el marco de plantación óptimo, que permita obtener mayores rendimientos sin depreciar la calidad del fruto. Para ello, se ha evaluado la producción tanto de las plantas hermafroditas como de las plantas femeninas durante dos ciclos de cultivo, así como las características organolépticas, grado de carpeloidía y deformación de los frutos. Los resultados indican que la densidad mayor, proporciona mejor comportamiento de las plantas así como mayor producción de frutos y menor porcentaje de fruta desechable.
\end{abstract}

Termos para indexação: Marcos de plantación, papaya, producción, frutos deformados

\section{DIFFERENT PLANTATION DENSITIES FOR PAPAYA (Carica papaya, L) CV. "BAIXINHO DE SANTA AMALIA" CULTURE IN THE CANARY ISLANDS}

ABSTRACT.- The growth of papaya plants under greenhouse conditions has recently become the norm in the Canary Islands. The use of "Baixinho de Santa Amalia" (a natural mutant of the "Sunrise" cultivar), which is a dwarf cultivar flowering early and not high up the plant, is the great interest, above all under these conditions. These phenological characteristic make possible the planting at higher densities than for other cultivars.

This study has been carried out to determine the optimal planting density to permit greater production without reducing fruit quality. To this aim the yield, both of hermaphrodite and female plants, has been evaluated through two crop cycles, as well as studying the organoleptic characteristics, degree of carpellody and deformation of the fruits. The results suggest that the highest density provides better plant perfomance as well as higher fruit production and lower percentage of rejected fruit.

Indes terms: plant spacing, papaya, production, fruit deformity

\section{INTRODUCCIÓN}

Las Islas Canarias situadas por debajo de los $30^{\circ}$ de latitud Norte, poseen en sus zonas de baja altitud clima de tipo subtropical que hace posible la adaptación de gran número de frutales tropicales. La papaya (Carica papaya, L.) se conoce en las islas desde hace más de 200 años y tradicionalmente el agricultor canario la ha cultivado en los bordes de la platanera o asociada a otros cultivos (Rodríguez et al.1995). La introducción de cultivares comerciales tipo "Solo" (Rodríguez, 1989) ha permitido el desarrollo del cultivo en los últimos 20 años. Actualmente, el nuevo cultivar "Baixinho de Santa Amalia" procedente de Brasil (Marín et al.1995) y de reciente introducción, ha generalizado su expansión sobre todo bajo condiciones de invernadero. La utilización de este cultivar (mutante natural del cv. "Sunrise") de porte bajo, longitud de hoja pequeña, emisión de la primera flor a corta altura y precocidad en la floración, resulta de gran interés especialmente en esta clase de cultivo protegido. Estas características fenológicas hacen posible el manejo de la plantación a mayores densidades que las empleadas con otros cultivares, (Nakasone et al. 1998) pero no impiden por otra parte, que se presenten formas aplastadas en los frutos debido a los entrenudos cortos que posee este cultivar, así como deformaciones propias de la carpeloidía. Por lo tanto, se ha llevado a cabo este trabajo cuyo objetivo ha sido determinar la densidad de plantación más adecuada para el cultivar "Baixinho de Santa Amalia" bajo cubierta de plástico, que permita obtener mayor rendimiento y menor proporción de fruta no comercial.

\section{MATERIALESYMÉTODOS}

Ensayo: Invernadero de plástico $\left(2.400 \mathrm{~m}^{2}\right)$

Duración del ensayo: 3 años (2 ciclos productivos) desde julio de 1996 hasta julio de 1999

Cultivar: "Baixinho de Santa Amalia" (mutante natural del cv. "Sunrise") procedente de semilla de EMCAPA (Brasil)
Localización: Finca "La Planta" (Güimar, Tenerife) perteneciente al Instituto Canario de Investigaciones Agrarias

Diseño estadístico: Plantas distribuidas irrestrictamente al azar. Unidad experimental: 1 planta

$\mathrm{N}^{\mathrm{o}}$ de tratamientos: T1 (2,5x1,5 m), T2 (2,5x2,5 m), T3 (2x2x3 m) y T4 (3x1,5 $\mathrm{m})$

$\mathrm{N}^{\circ}$ de repeticiones: según marcos de plantación:
a) T1: 24 plantas
c) T3: 28 plantas
b) T2: 35 plantas
d) T4: 24 plantas

Parámetros a medir:

Productividad: Kg./planta/sexo, $\mathrm{n}^{\mathrm{o}}$ de frutos/planta/sexo, peso medio/ planta/sexo, $n^{\circ}$ de frutos carpeloides/planta/sexo, $n^{\circ}$ de frutos aplastados /planta/sexo

Características descriptivas del fruto: longitud, anchura, longitud/ anchura, grosor de la pulpa, sólidos solubles totales y volumen de la cavidad

Para la evaluación de los parámetros sobre productividad se realizó un análisis de la varianza con un test de separación de medias de Fisher con $\mathrm{p}<0.05$. Para los parámetros referidos a las características descriptivas del fruto se realizaron observaciones de al menos 100 frutos por tratamiento y sexo y se realizaron las medias de los dos ciclos

\section{RESULTADOS Y DISCUSIÓN}

\section{Producción y Rendimientos}

Las plantas han entrado en producción rápidamente ( 9 meses después de la plantación), lo que pone de manifiesto su buena adaptación al medio, (Fig. 1). Las plantas hermafroditas y femeninas que pertenecen al marco de plantación más amplio (2,5 x 2,5 m), producen más kilos por planta que el resto, no existiendo diferencias significativas entre ciclos pero sí entre tratamientos. Hay que destacar que en el segundo ciclo, las plantas femeninas producen mayor número de kilos por planta que las hermafroditas, mientras que en las hermafroditas ocurre al contrario, el 
número de kilos es mayor en el primer ciclo, (Figs. 2 y 3 ). Sin embargo, el marco de plantación $(2,5 \times 1,5 \mathrm{~m})$ cuya densidad es mayor $(2.666 \mathrm{pl} / \mathrm{ha})$, produce mayor rendimiento por hectárea que los otros tratamientos tanto en el caso de las plantas hermafroditas como en las femeninas (Tablas 1 y 2), corroborando lo indicado por Arango et al.(1986). Se puede observar también en las mismas tablas, que el menor porcentaje de fruta no comercial (carpeloide y deforme por aplastamiento) corresponde igualmente al marco de plantación más denso (2,5 x 1,5 m).

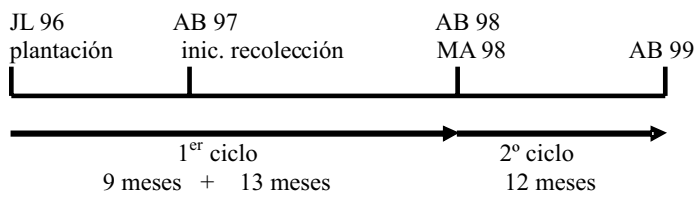

FIGURA 1 - Ciclos de producción del cv. "Baixinho de Santa Amalia". 1er ciclo (Plantación: julio 96. Recolección: abril 97 - abril 98). $2^{\circ}$ ciclo (continuación-Recolección: mayo 98 - abril 99).

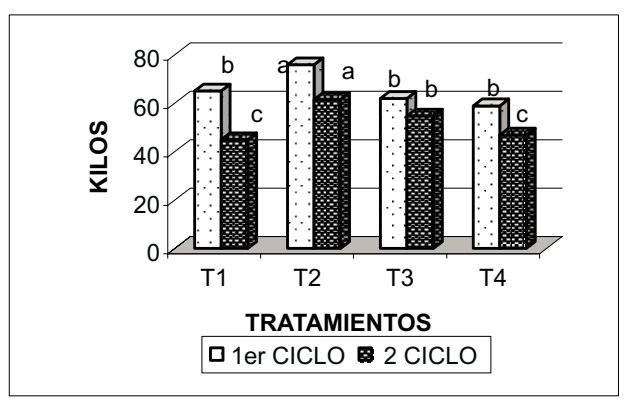

FIGURA 2 - Producción de frutos hermafroditas por planta y tratamiento en el $1^{\circ}$ y $2^{\circ}$ ciclo.

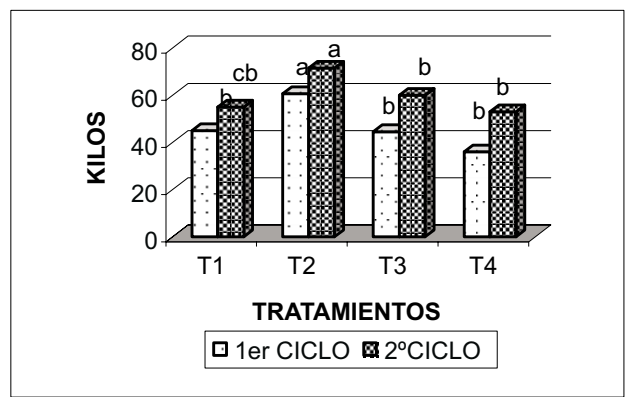

FIGURA 3 - Producción de frutos femeninos por planta y tratamiento en el $1^{\circ}$ y $2^{\circ}$ ciclo.

TABLA 1 - Rendimientos (kg/ha) de las plantas hermafroditas en dos ciclos productivos del cv. Baixinho de Santa Amalia bajo condiciones de invernadero en Islas Canarias.

\begin{tabular}{lccc}
\hline Densidades & $\mathrm{kg}$ totales & $\mathrm{kg}$ comerciales & $\mathrm{kg}$ no comerciales \\
\hline $2.666 / \mathrm{pl} / \mathrm{ha}$ & 293.822 & 244.768 & $17 \%$ \\
$1.600 / \mathrm{pl} / \mathrm{ha}$ & 220.160 & 164.160 & $25 \%$ \\
$2.000 / \mathrm{pl} / \mathrm{ha}$ & 233.600 & 164.600 & $30 \%$ \\
$2.222 / \mathrm{pl} / \mathrm{ha}$ & 235.320 & 173.104 & $26 \%$ \\
\hline
\end{tabular}

TABLA 2 - Rendimientos (kg/ha) de las plantas femeninas en dos ciclos productivos del cv. Baixinho de Santa Amalia bajo condiciones de invernadero en Canarias.

\begin{tabular}{llcc}
\hline Densidades & $\mathrm{kg}$ totales & $\mathrm{kg}$ comerciales & $\mathrm{kg}$ no comerciales \\
\hline $2.666 / \mathrm{pl} / \mathrm{ha}$ & 266.600 & 182.222 & $\% 32$ \\
$1.600 / \mathrm{pl} / \mathrm{ha}$ & 211.520 & 134.926 & $\% 36$ \\
$2.000 / \mathrm{pl} / \mathrm{ha}$ & 209.000 & 168.000 & $\% 19$ \\
$2.222 / \mathrm{pl} / \mathrm{ha}$ & 197.758 & 142.430 & $\% 28$ \\
\hline
\end{tabular}

\section{Número de frutos}

Según las figuras 4 y 5 , las plantas hermafroditas tienen menor número de frutos por planta que el resto de los tratamientos en el segundo ciclo y con el marco de plantación más pequeño $(2,5 \times 1,5 \mathrm{~m})$, tal como lo refieren Camejo et al.(1983), Bose et al.(1992), no ocurriendo así en el primer ciclo. Existiendo diferencias significativas entre las plantas del marco más denso y el resto, sólo en el segundo ciclo. Sin embargo, el número de frutos de las plantas femeninas del marco de plantación más denso (2,5 x 1,5 m) es menor que en el resto, tanto en el primer ciclo como en el segundo, corroborando una vez más lo probado por Camejo et al.(1983) y Reddy (1995) que indican que el número de frutos por planta es inversamente proporcional a la densidad de plantación aunque sólo se aprecie en este caso alguna diferencia significativa entre tratamientos.

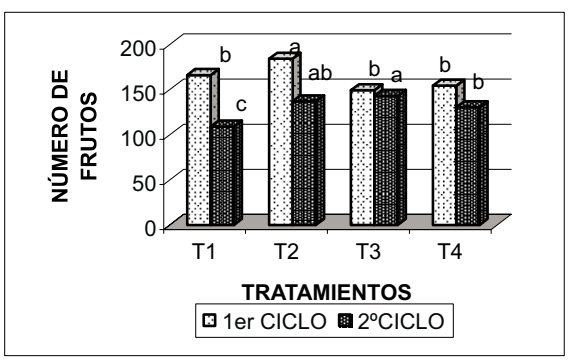

FIGURA 4 - Número de frutos hermafroditas por planta y tratamiento en el $1^{\circ}$ y $2^{\circ}$ ciclo.

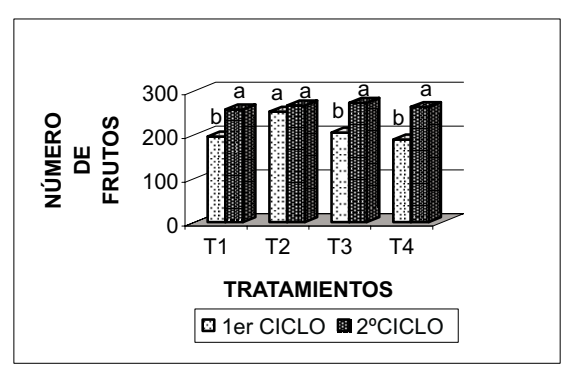

FIGURA 5 - Número de frutos femeninos por planta y tratamiento en el $1^{\circ}$ y $2^{\circ}$ ciclo.

\section{Peso del fruto}

El peso de los frutos hermafroditas y femeninos de las plantas pertenecientes al marco de plantación menos denso $(2,5 \times 2,5 \mathrm{~m})$ es mayor (Figs. 6 y 7) de acuerdo con lo mencionado por Kist et al.(1995), no existiendo diferencias significativas entre ciclos. Además destacar, que los frutos hermafroditas pesan siempre más que los frutos femeninos debido probablemente a que las flores hermafroditas se autopolinizan en un $99 \%$ de los casos (Chan et al.1994) y la posibilidad de fecundación es mayor que en las femeninas, las cuales necesitan de un vector (viento, insectos) para que la polinización se lleve a cabo. En este caso, la polinización ha sido deficiente o casi nula en el invernadero, (ausencia de ventilación suficiente) por lo que los frutos femeninos en su mayoría no han tenido semillas, han sido de menor tamaño y por lo tanto han pesado menos $(<300 \mathrm{~g})$. Por todo ello, es recomendable la siembra al menos de dos o más plantas por hoyo, para eliminar las femeninas una vez que hayan florecido y así lograr una mayor y mejor producción.

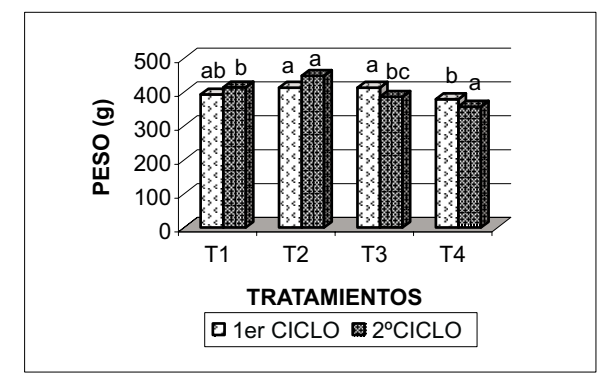

FIGURA 6 - Peso medio de los frutos hermafroditas por planta y tratamiento en el $1^{\circ}$ y $2^{\circ}$ ciclo. 


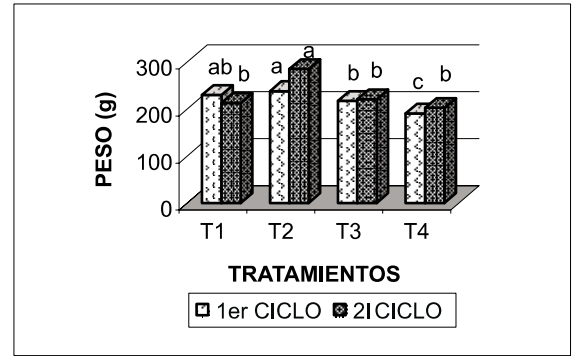

FIGURA 7 - Peso medio de los frutos femeninos por planta y tratamiento en el $1^{\circ}$ y $2^{\circ}$ ciclo.

\section{Número de frutos deformes}

El número de frutos deformes de las plantas hermafroditas en los dos ciclos y para cualquiera de los tratamientos es insignificante $(<6$ frutos/planta) (Fig. 8). Sin embargo, cuando se trata de las plantas femeninas el número se incrementa notablemente sobre todo en el segundo ciclo (Fig. 9). Teniendo en cuenta, que la producción es también mayor, como se ha visto anteriormente, se sugiere que tanto las condiciones favorables del invernadero como la propia estabilidad de la flor femenina podrían explicar estos resultados.

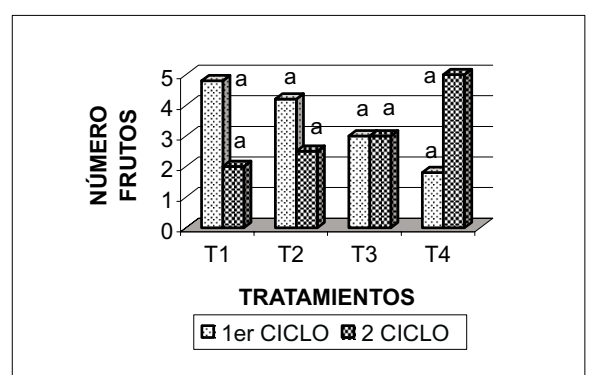

FIGURA 8 - Frutos hermafroditas aplastados por planta y tratamiento en el $1^{\circ}$ y $2^{\circ}$ ciclo.

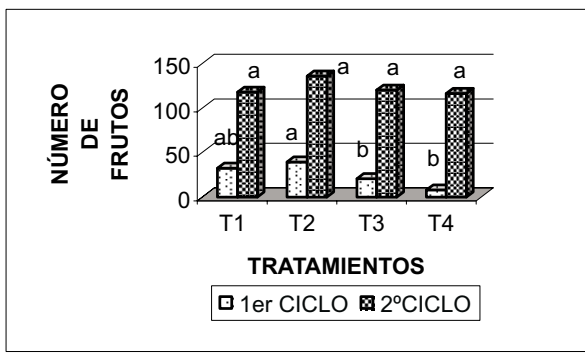

FIGURA 9 - Número de frutos femeninos aplastados por planta y tratamiento en el $1^{\circ}$ y $2^{\circ}$ ciclo.

\section{Número de frutos carpeloides}

De la figura 10 se deduce que, las plantas hermafroditas del marco de plantación menor $(2,5 \times 1,5 \mathrm{~m})$ y por lo tanto con mayor densidad (2.666 pl/ha), tienen menor número de frutos carpeloides que el resto de los tratamientos, tanto en el primer ciclo como en el segundo, existiendo diferencias significativas de este tratamiento con el resto de ellos. Es posible que la orientación del invernadero haya influido en este sentido y por lo tanto un aumento de la temperatura en esa zona. Se destaca también, que la disminución de la carpeloidía en el segundo ciclo con respecto al primero, posiblemente sea debida al incremento en la edad de las plantas (Nakasone et al. 1998). Los frutos femeninos por el contrario no presentan este fenómeno ya que las flores de las que proceden son estables respecto a las condiciones ambientales (Storey, 1958), como ya se ha mencionado.

\section{Características descriptivas de los frutos hermafroditas y femeninos}

Los frutos hermafroditas y femeninos presentan características descriptivas muy semejantes entre ellos (Tablas 3 y 4), destacando los mayores porcentajes de sólidos solubles totales (T.S.S.) en los frutos hermafroditas, de forma contraria a lo que ocurre normalmente y dentro de los límites requeridos en el mercado de exportación 11,5\% \% Brix (Nakasone et al. 1998).

A la vista de estos resultados hay que señalar que en Canarias, las plantas hermafroditas son más adecuadas para el cultivo de papaya en invernadero que las femeninas, ya que las flores procedentes de plantas hermafroditas (cleistógamas) no tendrán problemas de polinización y el porcentaje de carpeloidía es bajo. Además, otra de las ventajas a señalar es que el agricultor podrá obtener sus propias plantas a partir de las semillas obtenidas por autopolinización previo ensacado de las flores antes de la antesis, lo que supondrá un considerable ahorro económico.

Por otra parte, los frutos piriformes son más apreciados en el mercado de exportación que los femeninos. Así, habrá que considerar que los trabajos que se realicen en un futuro, deberán estar orientados en este sentido y con cultivares de porte bajo como el referido en este trabajo u otros como el cv. BH-65 procedente de Sudáfrica recientemente introducido en Canarias.

TABLA 3 - Características descriptivas de los frutos hermafroditas del cv. Baixinho de Santa Amalia bajo condiciones de invernadero en Islas Canarias.

\begin{tabular}{|c|c|c|c|c|c|c|}
\hline Tratamientos & $\begin{array}{c}\text { Longitud } \\
(\mathrm{cm})\end{array}$ & $\begin{array}{c}\text { Anchura } \\
(\mathrm{cm})\end{array}$ & $\begin{array}{l}\text { Long./Anch. } \\
\text { (cm) }\end{array}$ & $\begin{array}{c}\text { Grosor pulpa } \\
(\mathrm{cm})\end{array}$ & T.S.S. ${ }^{\circ}$ Brix & $\begin{array}{c}\text { Vol./Cav } \\
\text { (cc) }\end{array}$ \\
\hline $\mathrm{T} 1(2,5 \mathrm{x} 1,5 \mathrm{~m})$ & 13,63 & 8,20 & 1,66 & 1,90 & 11,30 & 84,13 \\
\hline $\mathrm{T} 2(2,5 \times 2,5 \mathrm{~m})$ & 14,14 & 8,40 & 1,68 & 2,00 & 11,67 & 83,75 \\
\hline $\mathrm{T} 3(2 \times 2 \times 3 \mathrm{~m})$ & 13,89 & 8,25 & 1,68 & 1,92 & 11,16 & 84,31 \\
\hline $\mathrm{T} 4(3 \mathrm{x} 1,5 \mathrm{~m})$ & 14,82 & 8,07 & 1,84 & 1,89 & 11,45 & 79,54 \\
\hline
\end{tabular}

TABLA 4 - Características descriptivas de los frutos femeninos del cv. Baixinho de Santa Amalia bajo condiciones de invernadero en Islas Canarias.

\begin{tabular}{lcccccc}
\hline Tratamientos & $\begin{array}{c}\text { Longitud } \\
(\mathrm{cm})\end{array}$ & $\begin{array}{c}\text { Anchura } \\
(\mathrm{cm})\end{array}$ & $\begin{array}{c}\text { Long./Anch. Grosor pulpa } \\
(\mathrm{cm})\end{array}$ & $\begin{array}{c}\text { T.S.S. }{ }^{\circ} \text { Brix } \\
(\mathrm{cm})\end{array}$ & $\begin{array}{c}\text { Vol./Cav } \\
(\mathrm{cc})\end{array}$ \\
\hline T1 $(2,5 \times 1,5 \mathrm{~m})$ & 9,98 & 7,32 & 1,36 & 1,53 & 10,57 & 55,00 \\
T2 $(2,5 \times 2,5 \mathrm{~m})$ & 10,35 & 7,55 & 1,37 & 1,61 & 1,551 & 55,91 \\
T3 $(2 \times 2 \times 3 \mathrm{~m})$ & 10,30 & 7,50 & 1,37 & 1,70 & 10,95 & 53,00 \\
T4 $(3 \times 1,5 \mathrm{~m})$ & 9,82 & 7,00 & 1,40 & 1,48 & 10,90 & 49,66 \\
\hline
\end{tabular}

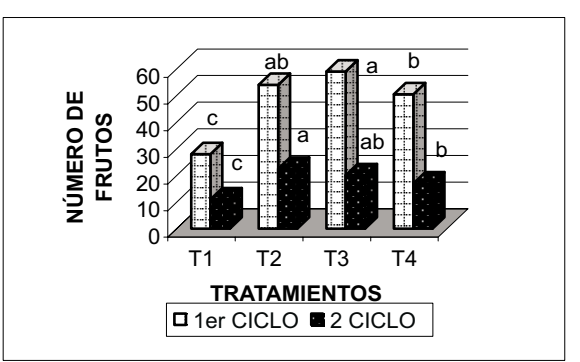

FIGURA 10 - Número de frutos hermafroditas carpeloides por planta y tratamiento en el $1^{\circ}$ y $2^{\circ}$ ciclo.

\section{Plagas y enfermedades}

Las principales plagas y enfermedades que incidieron en el cultivo fueron:

Araña roja (Tetranychus cinnabarinus), ataca a las hojas produciendo pequeñas y redondas manchas amarillas. La incidencia es mayor en verano.

Taladro (Opogona sacchari), se instala en las heridas producidas al caerse las hojas, una vez que se inicia la recolección de la fruta. Actualmente, esta plaga representa un problema importante en el cultivo de papaya en Canarias, pues resulta difícil su control.

Oídio (Oidium caricae), es muy frecuente a lo largo del año, pero 
incide con mayor agresividad en época de alta humedad. Ha sido controlado con relativa facilidad. Ataca a las hojas principalmente.

Botritis (Botritis theobromae), este hongo aparece de forma esporádica en las plantas de papaya en invierno, en las zonas más húmedas del invernadero donde existe un marco de plantación más pequeño.

Finalmente, otro problema de escasa importancia ( sólo una planta se vio afectada) surgido durante el cultivo ha sido la aparición de la enfermedad de causa desconocida llamada "colapso de la planta o muerte súbita". Repentinamente, las hojas de la planta se debilitan, se inclinan hacia abajo adhiriéndose al tallo y posteriormente caen al suelo. Esta enfermedad se cree estar asociada a suelos encharcados y pobres en drenaje.

\section{CONCLUSIONES}

Se puede concluir diciendo que en las plantas hermafroditas, el tratamiento de mayor densidad T1 (2.666 pl/ha) es el que produce mayor rendimiento y menor porcentaje de fruta no comercial. A su vez, las plantas femeninas producen en general frutos con poco peso, mal polinizados y deformados en cualquiera de los tratamientos y principalmente en el segundo ciclo, por lo tanto, es recomendable no cultivar este tipo de plantas o eliminarlas en el momento de la floración.

\section{REFERENCIASBIBLIOGRÁFICAS}

ARANGO, W. L. V.; BEDOYA, V.L. Y SALAZAR, C. R.. Determinación de distancias de siembra en papayo, Carica papaya, L. para la zona plana del Valle del Cauca. Acta Agronómica, Palmira,v.36, n.1, p.3444,1986

BOSE, T. K.; MITRA, S. K. Y CHATTOPADHYA, Y. Optimum plant density for some tropical fruit crops. Acta Horticulturae, Wageningen, v.296, p.171-176,1992

CAMEJO, B. Y ALVAREZ, P.R. Efecto de la densidad de plantación en papaya (Carica papaya, L.) cultivar "Maradol Rojo". Ciencias Técnicas Agrícolas. Cítricos y otros frutales, v.5,n.3, p.69-79, 1983.

CHAN,Y. K.; UTHAI, N.; WISNU, B.; KOAY, S. H. AND ESPINO, R.C. Commercial papaya cultivars in ASEAN. In: ROHANI Md. Yon. Papaya. Malasia: Ed. Food Technology Research Centre, 1994. p.5-17

KIST, H. Y MANICA, I. Mamoeiro: densidades de plantio. Cuadernos de Horticultura UFRGS, v.5, p.1-10,1995

MARÍN, S.L. D.; GOMES, J.A.; SALGADO, J.S.; MARTINS, D. S. Y FULLIN, E. RecomendaVoes para a cultura do mamoeiro dos grupos Solo e Formosa no Estado do Espirito Santo 4.Vitória, Es: Emcapa, 1995. p.16-17

NAKASONE, H. Y. AND PAULL, R. E. Papaya. In: Tropical fruits. New York: Ed. Cab International, 1998. p.239-269

REDDY, Y. T. N. Effect of plant spacing on "Coorg Honey Dew" papaya (Carica papaya, L.). Indian Journal of Agricultural Sciences, New Delhi, v.65, n.2, p.130-132, 1995

RODRÍGUEZPASTOR, M. C. Estudio sobre la fenología, comportamiento floral y características del fruto de papaya (Carica papaya, L.) en varios cultivares del grupo "Solo", con especial referencia a la carpeloidía, en las condiciones canarias. 1989. 280 f. Tesis (Doctora en Biología). - Universidad de La Laguna, Facultad de Biología, 1989.

RODRÍGUEZ PASTOR, M. C.; GALÁN SAÚCO, V. Y ESPINO DE PAZ, A. I. Técnicas de cultivo de papaya en Canarias. Canarias: Consejería de Agricultura y Alimentación, 1995. p.23 (Cuaderno de Divulgación 1/95)

STOREY, W. B. Modification of sex expression in papaya. Horticultural Advance, Saharanpur, n.2 p.49-60,1995 